\title{
Study on noise reduction of nozzle shock diamond flow destroyed by flexible components
}

\author{
Chung-Hwei Su${ }^{1}$, Chien-Chih Chen ${ }^{2}$, Yi-Hua $\operatorname{Pan}^{3}$, Chen-Ching Ting ${ }^{4}$, * \\ ${ }^{1}$ Department of Safety, Health and Environmental Engineering, National Kaohsiung First University of Science and Technology, Taiwan \\ ${ }^{2}$ GraduateInstituteof Mechanical and Electrical Engineering, National Taipei University of Technology, Taipei, 10608 Taiwan \\ ${ }^{3}$ Institute of Mechatronic Engineering, National Taipei University of Technology, Taipei, 10608 Taiwan \\ ${ }^{4}$ Department of Mechanical Engineering, National Taipei University of Technology, Taipei, 10608 Taiwan
}

\section{Email address:}

such@nkfust.edu.tw (Chung-Hwei Su),t8669030@ntut.edu.tw (Chien-Chih Chen), t101508029@ntut.edu.tw (Yi-Hua Pan), chchting@ntut.edu.tw (Chen-Ching Ting)

\section{To cite this article:}

Chung-Hwei Su, Chien-Chih Chen, Yi-Hua Pan, Chen-Ching Ting. Study On Noise Reduction of Nozzle Shock Diamond Flow Destroyed by Flexible Components. American Journal of Optics and Photonics. Vol. 2, No. 2, 2014, pp. 12-17. doi: 10.11648/j.ajop.20140202.11

\begin{abstract}
It's well known that the high pressure flow running through the nozzle yields shock diamond flow which mainly causes the noise. This work employed flexible components on the nozzle outlet to destroy shock diamond flow and using micro color schlieren technique to visual the dispersion shock diamond. Experiments adjusted conditions of and mesh flexible components for optimal design. The results show that wire diameter $0.5 \mathrm{~mm}$ of cross flexible component, away from nozzle $0.5 \mathrm{~cm}$ and $1 \mathrm{~cm}$ received the noise of $83.8 \mathrm{~dB}$, the reducing rate was ca. $12.5 \%$. The mesh 100 flexible component away from nozzle $0.5 \mathrm{~cm}$ received the noise of $75.6 \mathrm{~dB}$, the reducing rate was ca. $21.17 \%$.
\end{abstract}

Keywords: Nozzle Noise, Nozzle Flow, Micro Color Schlieren, Shock Diamond Flow

\section{Introduction}

Sound noise always exists in people's daily life, the appropriate sound of energy and frequency can purify people's hearts, optimizing social and create artistic value, such as music, lectures and so on. However, excessive and inappropriate frequency sound for human beings is a noise. Noise damage on human hearing according to their severity is divided into "temporary hearing loss" and "permanent hearing loss" two kinds. The "temporary hearing loss" is the loss of human hearing brief, that the sound can't be interpreted by auditory nerve damage. The "Permanent hearing loss" is caused by auditory nerve receptors or permanent hearing damaged [1-2].

The "Permanent hearing loss" is cause by auditory receptors or auditory receptors permanently injured. The long and numerous "temporary hearing loss" will accumulate into a "permanent hearing loss"[3-5]. Noise is the voice of physical energy, it generate by auditory receptors and auditory nerve on the body, not only to detrimental the ears of the hearing, through the nervous system generate non-auditory effects on the human body. When exposed to noise decibels above $90 \mathrm{db}$ for several hours, it will produce a temporary hearing loss[6-7].
Long-term exposure to loud noise or high volume, it will cause hearing loss even if the time is very short. Noise will affect sleep, hinder conversation, low working efficiency, disgust, anger and other psychological effects, over time, due to psychological reactions, insomnia caused by physiological dysfunction of the phenomenon, such as headache, dizziness, inability to concentrate both direct and indirect impact of noise [8-10], it will adopt a inaudible noise to make their own coping strategies to combat environmental "noise", this will result in a noisy environment where children become adverse effects of inadvertently sound signal, especially children, is not easy to distinguish between the importance of sound, children will be learning and cognitive development have a considerable impact [11-15].

Schlieren optical technique in the field of engineering and the physical sciences is a very basic and valuable optical measurement methods, it's often used to obtain a transparent medium in the two-dimensional distribution of the refractive index gradient. More focuses on ventilation, shockwave of wind tunnel experiments, nozzle sound pressure distribution and flow field for air condition[16-18].

Schlieren optics also is a powerful technique to study 
noise problems, sound waves are longitudinal waves, but also the mechanical waves, discussion on the volatility, the sound wave and light wave substantially the same. Acoustic wave and light waves in properties of the performance differences are must be passed through the media. Transfer process, the medium density will generate changes in density. If it can image transfer status of change the density in the medium, research of noise space will be the most direct. Sound field imaging can use optics technology on the transfer medium density development.

Study of shock diamond flow destruction of optical flow is quite rare, therefore, In this study, design the different forms of the nozzle exit, and using the micro color schlieren technique to observe change of shock diamond flow, and then reducing the noise generated of the nozzle,

It's well known that the high pressure flow running through the nozzle yields shock diamond flow which mainly causes the noise. This work employed flexible components on the nozzle outlet to destroy shock diamond flow and using micro color schlieren technique to visual the dispersion shock diamond. Experiments adjusted conditions of and mesh flexible components for optimal design. The results show that wire diameter $0.5 \mathrm{~mm}$ of cross flexible component, away from nozzle $0.5 \mathrm{cmand} 1 \mathrm{~cm}$ received the noise of $83.8 \mathrm{~dB}$, the reducing rate was ca. $12.5 \%$. The mesh 100 flexible components away from nozzle $0.5 \mathrm{cmreceived}$ the noise of $75.6 \mathrm{~dB}$, the reducing rate was ca. $21.17 \%$.

\section{Theory}

The applications of micro color schlieren technology is relationship with fluid density $(\rho)$ and index of refractionn.

It's relationship formula proposed by the Gladstone and Dale.

$$
\mathrm{n}-1 \propto \rho
$$

Gladstone and Dale Further import value of the constant $\mathrm{K}$, and obtain

$$
\mathrm{n}-1=\mathrm{k} \rho
$$

Formula (2) is called Gladstone-Dale Equation, constant $\mathrm{K}$ is called the Gladstone-Dale constant, the units of $\mathrm{K}$ is $\mathrm{cm}^{2}$, value $\mathrm{K}$ varies with the wavelength. By the ideal gas equation

$$
\mathrm{p}=\rho \mathrm{RT}
$$

Among, $\mathrm{P}$ is the pressure, $\mathrm{R}$ is the ideal gas constant, $\mathrm{T}$ is the temperature, so the constant temperature can be obtained formula (4).

$$
\rho \propto P
$$

According to Gladstone-Dale relationship proving the formula (5)

$$
n-1 \propto P
$$

Micro color schlieren technique can immediately observe phenomena of fluid flow by changes in fluid density or temperature. When the area of parallel light with a fluid flowing, the density of the fluid will change, therefore, the traveling direction of light were changed, so that generate an angle of deflection of light. Deflection of light through a second lens intersect arrive focal point, adjust the position of Knife-Edge, By blocking out the deflection larger of light. The screen imaging when the deflection smaller of light exposure to the screen, image on the screen is the immediate images of fluid flow, this flowing fluid must be transparent. When light passes through the flow of field non-uniform density, it will cause deflection of the phenomenon, this phenomenon is due to change the refractive index of light [19].

\section{Experimental Setup}

This paper using cross- type and mesh-type flexibility material to reduce noise of high-pressure nozzle, and use micro color schlieren technique to observe airflow. Experiment divided into three parts to introduction, erection of micro color schlieren, Design the flexible material nozzle to damage compression waves and measurement noise of nozzle.

Micro color schlieren use Z-type optical path arrangement. Fig. 1showsschematic of the micro color schlieren setup which uses two $15 \mathrm{~cm}$ diameter of concave mirror(f/6) to generate parallel light. While the fluid go through the area of parallel light, the light density of the fluid will be changed. Therefore, the traveling direction of light were changed. The screen will show a different degree of color images. By adjusting the aperture size in top right of erection schematic cover a larger angle of light which can control image sensitivity of the micro color schlieren image. Finally, the image recorded by camera memory card, which can observe the fluid flow behavior in the camera.

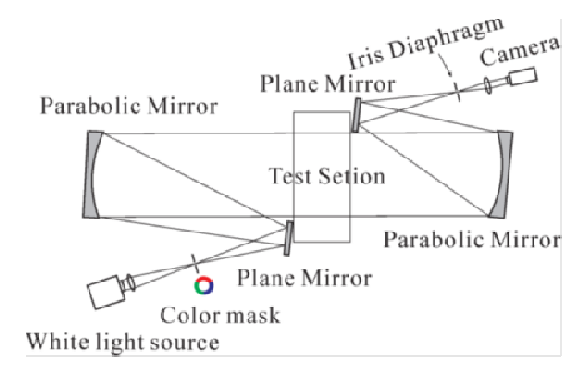

Figure 1. Schematic of the micro color schlieren setup

Design of flexible nozzle is divided into flexible material of cross-type and mesh-type. Cross-type flexible material of stainless steel wire under diameter 0.2,0.3,0.4,0.5,0.6 mm. The test conditions by adjusting flexible material distance from the nozzle exit, the distance of the flexible material from the nozzle exit $0,0.5,1,1.5,2,2.5,3 \mathrm{~cm}$.Fig. 2 is the physical map of nozzle compression wave damage by cross flexible material, flexible mesh material used stainless steel mesh, whose grid mesh net has $14,40,60,80,100$, the 
distance of the flexible material from the nozzle exit $0,0.5,1,1.5,2,2.5,3 \mathrm{~cm}$.Fig.3is the physical map of nozzle compression wave damage by flexible mesh material.

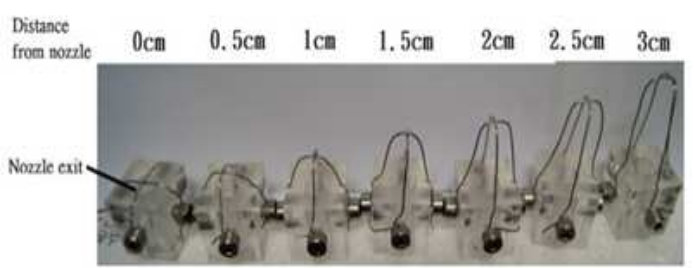

Figure 2.Nozzle compression wave damage by cross flexible material

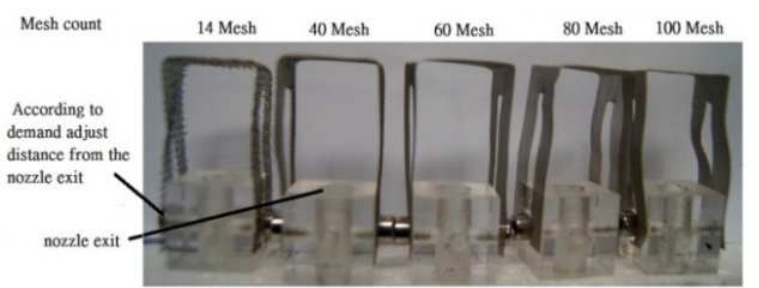

Figure 3.Physical map of nozzle compression wave damage by flexible mesh material.

Under measurements noise of nozzle, this article experimental test conditions and environmental needs follows the CNS 3805-C4127 specification[19]. Fig.4 is the measurement of nozzle noise diagram. Fig. 5 is the physical map of measurement noise nozzle. Test environment is a free sound field, select A-weighted sound level meter in this case.

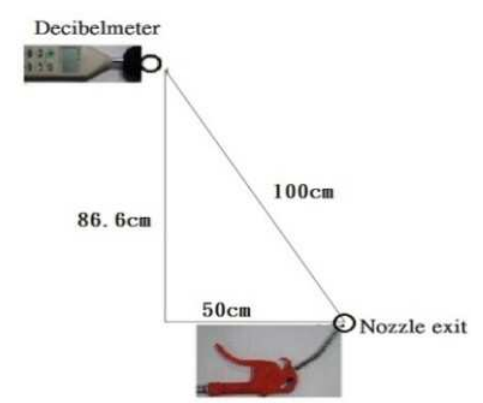

Figure 4.Measurement of nozzle noise diagram

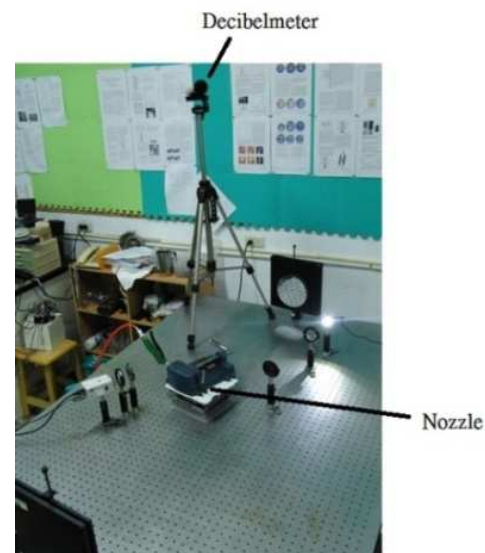

Figure 5.Physical map of measurement noise nozzle.

\section{Results and Discussion}

In this study, the experiments reduction noise nozzle flow, experiments with compressed gas as the working medium to explore, and design a variety of different types of nozzle form, with Micro color schlieren technique to observe scenario of the various forms of fluid flow in nozzle exit, and using decibels, measured the noise value (dB). Experimental observations have a nozzle flow imaging of general commercial nozzle and flexible material nozzle, nozzle exit diameter is $4 \mathrm{~mm}$.

Experimental observation nozzle flow imaging of the general commercial at first, and measurements noise value, Fig.6 shows the flow situation of normal commercial air injection nozzles distribute the shock diamond flow, noise is $95.8 \mathrm{~dB}$. This experiment will be made flexible material nozzles, to reduce the general commercial nozzle noise.

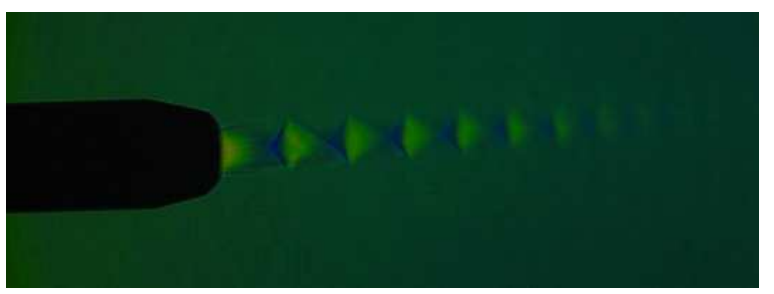

Figure6. Flow image of commercial general nozzle.

The nozzle of flexible material is mainly used cross-type nozzle and mesh-type flexible material to destroy shock diamond flow, with the same wire diameter and changes the distance from the nozzle exit, Fig.7 shows the cross-type flexible material nozzle results in comparison chart of noise level. Fig. 8 (a) (g) is the micro color schlieren imaging, the best parameters of each wire diameter distance from the nozzle exit. Among them, the distance from the nozzle exit is $0.5 \mathrm{~cm}$,wire diameter is $0.5-1 \mathrm{~mm}$ show the best parameters Fig. 8 (a)(b), when the distance from nozzle exit is $0 \mathrm{~cm}$, the airflow is divided into four bundles, the cross flexible material distance farther, the results of noise reduction are worse, because the compressed shock diamond flow is not to destruction by cross line, which distance from the exit is $0-1 \mathrm{~cm}$ was best.

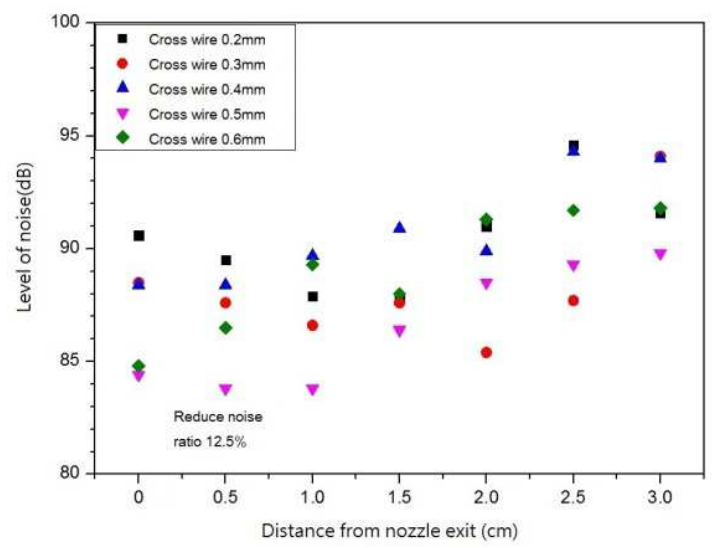

Figure 7.Cross-shaped flexible material nozzle results in comparison chart of noise level. 
In part of silencer method of mesh type nozzle, change the mesh count of stainless steel woven mesh and distance from nozzle exit to observation of effect of wave impact. Fig.9 shows the mesh-type flexible material nozzle results in comparison chart of noise level. Fig. 9shows the micro color schlieren imaging, the best parameters of each mesh count distance from the nozzle exit. Among them, the number

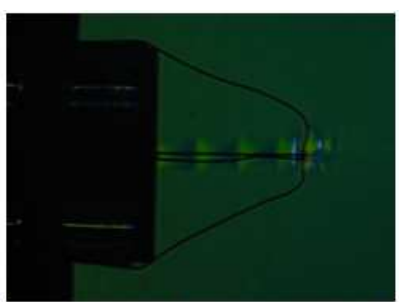

(a) Wire diameter is $0.2 \mathrm{~m}$, distance from nozzle exit is $1.5 \mathrm{~cm}$.

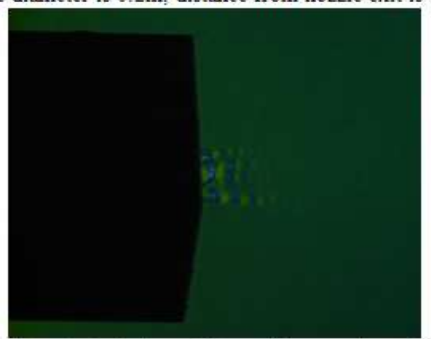

(c) Wire diameter is $0.4 \mathrm{~mm}$, distance from nozzle exit is $0 \mathrm{~cm}$.

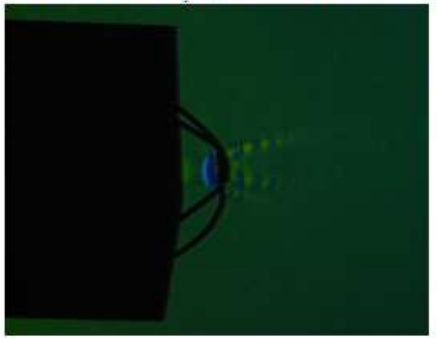

(e) Wire diameter is $0.5 \mathrm{~mm}$, distance from nozzle exit is $0.5 \mathrm{~cm}$

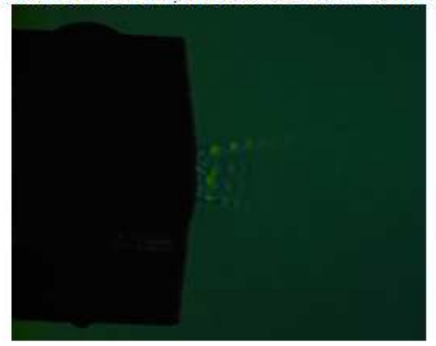

Figure8. Micro color schlieren imaging, the best parameters of each wire diameter distance from the nozzle exit.

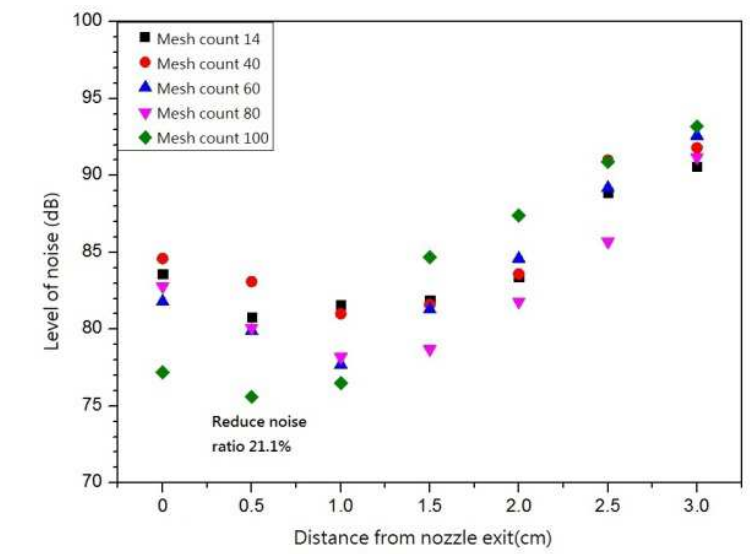

Figure9. Mesh-type flexible material nozzle results in comparison chart of noise level. mesh of 100-mesh, distance from the nozzle exit is $0.5 \mathrm{~mm}$ Fig.10(e), which is the best design parameters. According to the experimental results, the mesh count is larger, silencing effect is better. But when the number of mesh to 100 mesh, due to the larger mesh count, stainless steel pores are too small to generate flow resistance.

(b) Wire diameter is $0.3 \mathrm{~mm}$, distance from nozzle exit is $2 \mathrm{~cm}$.

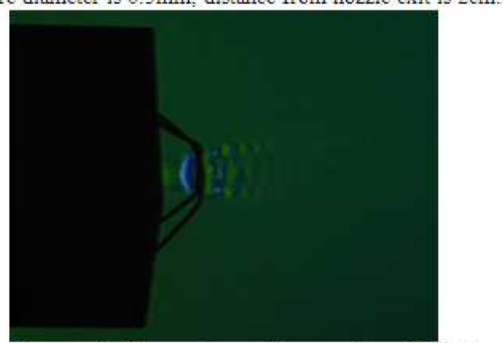

(d) Wire diameter is $0.4 \mathrm{~mm}$. distance from nozzle exit is $0.5 \mathrm{~cm}$

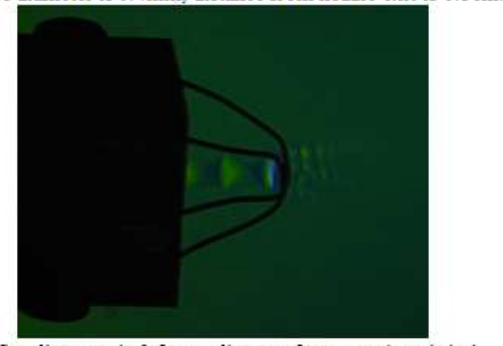

(f) Wire diameter is $0.5 \mathrm{~mm}$, distance from nozzle exit is 1

(g) Wire diameter is $0.6 \mathrm{~mm}$, distance from nozzle exit is $0 \mathrm{~cm}$ 


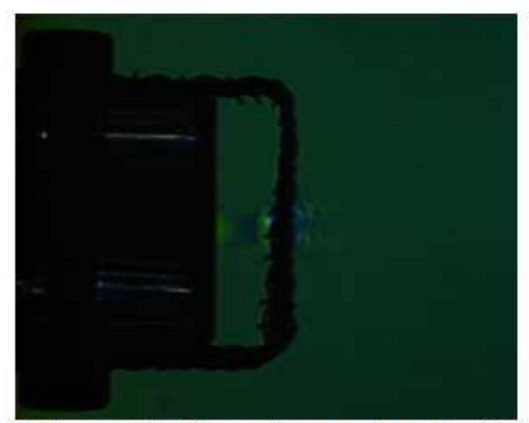

(a) Mesh count 14, distance from nozzle exit is $0.5 \mathrm{~cm}$.

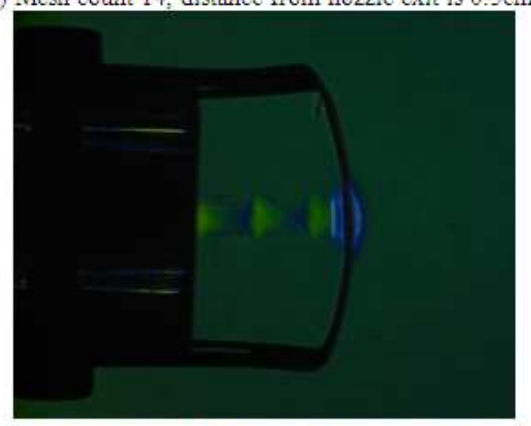

(c) Mesh count 60 , distance from nozzle exit is $1 \mathrm{~cm}$.

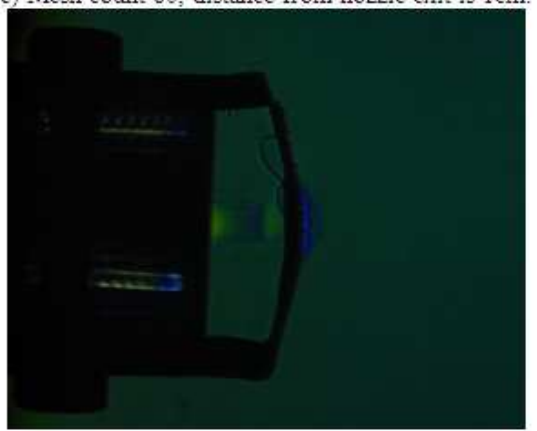

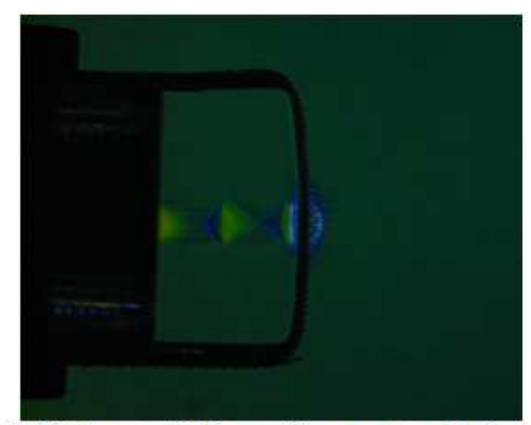
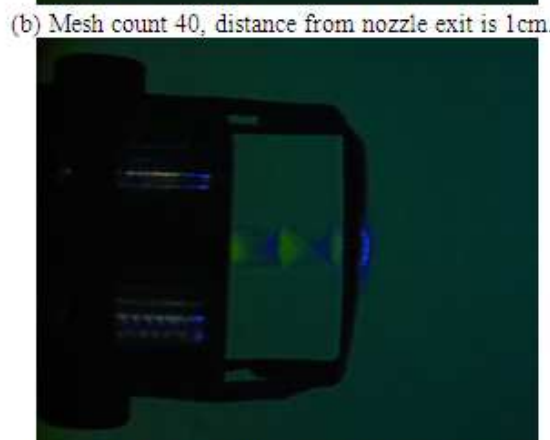

(d) Mesh count 80 , distance from nozzle exit is $1 \mathrm{~cm}$.

(e) Mesh count 100 , distance from nozzle exit is $0.5 \mathrm{~cm}$.

Figure 10.Micro color schlieren imaging, the best parameters of each mesh count distance from the nozzle exit.

\section{Conclusions}

This thesis employed micro color schlieren technique to observation high-pressure nozzle flow effects of noise reduction, the nozzle divided into Cross-type and mesh type flexible material. The results showed that, the part of cross-type flexible material nozzle, the noise from the original value $95.8 \mathrm{~dB}$, reduced to $83.8 \mathrm{~dB}$, the noise reduction value $12.0 \mathrm{~dB}$, the best of noise reduction rate about $12.5 \%$. Among, used a cross-type flexible material nozzle wire diameter was $0.5 \mathrm{~mm}$ and distance from nozzle exit was $0.5 \mathrm{~cm}$ and $1 \mathrm{~cm}$. The part of mesh-type flexible material nozzle. The noise from the original value $95.8 \mathrm{~dB}$, reduced to $75.6 \mathrm{~dB}$, the noise reduction value $20.2 \mathrm{~dB}$, the best of noise reduction rate about $21.1 \%$, Among, used meshtype of flexible material nozzle mesh count was $0.5 \mathrm{~mm}$ and distance from nozzle exit was $0.5 \mathrm{~cm}$. The mesh type nozzle biggest difference of cross type is compressed shock diamond flow of nozzle through the stainless steel wire mesh, there are effect of filtering, and generate spherical wave airflow diverging outward, and using the cross-type nozzle, the airflow will divided into four column, these two nozzle design successfully achieved effect of noise reduction.

\section{Acknowledgements}

The authors would like to acknowledge the financial support from the National Science Foundation of Taiwan under Grant No. NSC 102-2221-E-027-036.

\section{References}

[1] K. Lim and C. Lee, "A numerical study on the characteristics of flow field, temperature and concentration distribution according to changing the shape of separation plate of kitchen hood system," Energy and Buildings, vol. 40, 2008, pp. 175-184.

[2] Y. Li and A. Delsante, "Derivation of capture efficiency of kitchen range hoods in a confined space," Building and Environmental, vol. 31, no. 5, 1996, pp. 461-468.

[3] T. J. Liu and M. S. Young, "Eliminating contaminants with a piezoelectric transducer in the design of low-cost smart kitchen range hoods," IEEE Sensors Journal, vol. 2, no.4, 2002, pp. 314-321.

[4] J. Abanto and M. Reggio, "Numerical investigation of the flow in a kitchen hood system," it Building and Environment, vol. 41, 2006, pp. 288-296. 
[5] C. M. Chiang, C. M. Lai, P. C. Chou and Y. Y. Li, "The influence of an architectural design alternative (transoms) on indoor air environment in conventional kitchens in taiwan," Building and Environment, vol. 35, 2000, pp. 579-585.

[6] Q.F. Lin, "Design and application of "industrial ventilation," Environmental Progress, vol. 56, 2002.

[7] E. Gonzalez, F. Marzal, A. Minana and M. Doval, "Influence of exhaust hood geometry on the capture efficiency of lateral exhaust and push-pull ventilation systems in surface treatment tanks," Environmental Progress, vol. 27, 2008, pp. 405-411.

[8] B. Lishman and A. W. Woods, "The effect of gradual changes in wind speed or heat load on natural ventilation in athermally massive building," Building and Environment, vol. 44, 2009, pp. 762-772.

[9] N. T. Chen, C. C. Chiang, C. P. T. Chou, Y. P. Lin and Z. L. Chen, "Bedroom opening fitted vertical louvers on the impact of indoor natural ventilation," Architectural Journal,61, 2007, pp.63-78.

[10] Q. Q. Chen, "Kitchen transom hole high rate of carbon dioxide concentration field and the effect of ventilation rate," Master's thesis, National Cheng Kung University, Tainan, 2000.

[11] H. Y. Chen, "Theory of design exclusion pollution in plant," Master's thesis, Department of Refrigeration, Air Conditioning and Energy Engineering, Tainan, 2007.
[12] Z. X. Ma, "Blowing suction Industrial Ventilation hood numerical simulation of turbulent diffusion," master's thesis, Department of Mechanical Engineering, National Taiwan University of Technology, Taipei, 2005.

[13] W. X. Yuan, "Industrial Ventilationto improve air quality operating environment easy way" Environmental Progress,vol.12, 1995.

[14] J. H. Cheung, "Industrial Ventilationoperating environmental control of the weapon," Environmental Progress, vol.12, 1995.

[15] G. S. Settles, "Color schlieren optical-a review of techniques and application," Prinseton University, Prinseton, 1982.

[16] G.E.A. Meier, W.C. Selerowicz, and A.P. Szumowski, "A nozzle generating low jet noise," Journnl of Sound and Vibration," vol. 136, no. 1, 1990, pp. 65-73.

[17] C.C. Chen and C.C. Ting, "Investigating the effect of color mask on sensitivity for the color schlieren imaging," International Journal of Engineering and Technology Innovation, vol.3, no. 2 , 2013, pp. 114-122.

[18] C.C. Ting and C.C. Chen, "Detection of Gas Leakage Using Micro Color Schlieren Technique," Measurement, vol. 46, 2013, pp. 2467-2472.

[19] Chinese National Standard, CNS 3805. 